\title{
OFDM PAPR Reduction Using Clipping with Distortion Control
}

\author{
Shang-Kang Deng \\ Graduate Institute of Communication Engineering \\ National Taiwan University \\ Taipei 106, Taiwan, R.O.C. \\ Email: d88942008@ntu.edu.tw
}

\author{
Mao-Chao $\operatorname{Lin}^{\dagger}$ \\ Department of Electrical Engineering \\ National Taiwan University \\ Taipei 106, Taiwan, R.O.C. \\ Email: mclin@cc.ee.ntu.edu.tw
}

\begin{abstract}
We propose a new PAPR reduction scheme for OFDM systems. The idea is to reduce PAPR and control clipping distortion simultaneously. The procedure includes setting a proper distortion bound and recursive operations of clipping, filtering and distortion control. The proposed scheme can achieve significant PAPR reduction while maintaining low error rate. For this scheme, PAPR reduction is obtained without any redundancy and no side information is needed in the OFDM receiver. Hence, OFDM systems using this scheme do not pay the price of the reduction of transmission rate or reliability for achieving PAPR reduction.
\end{abstract}

Keywords - PAPR, recursive clipping and filtering, OFDM

\section{INTRODUCTION}

OFDM is a multicarrier modulation technique for broadband communication, the advantage of which includes the strong immunity to multipath fading, high spectral efficiency and easy compensation for frequency-selective channel [1]. However, a major disadvantage of OFDM is the inherent high peak-toaverage power ratio (PAPR) due to its approximately Gaussian distributed waveform generated by the summation of many subcarrier-modulated signals.

An OFDM system with high PAPR requires a large dynamic range for the power amplifier of the transmitter. Alternatively, power back-off in the amplifier can be used [2]. To avoid operating the amplifiers with large back-off, we must allow occasional saturation of power amplifiers or clipping the signal before feeding to the amplifier. Such arrangement will cause signal distortion and power spectral expansion [2], [3], [4]. Many PAPR reduction methods have been proposed. Some methods are designed based on employing redundancy, such as coding [4], [5], selective mapping with explicit or implicit side information [6], [7], [8], or tone reservation [9], [10]. An apparent effect of using redundancy for PAPR reduction is the reduced transmission rate. PAPR reduction may also be achieved by using extended signal constellation, such as tone injection [9], [11] or multi-amplitude CPM [12]. The associated drawback is the increased power and implementation complexity. A simple PAPR reduction method can be achieved by clipping the time-domain OFDM signal [2], [13], [14], [15]. The resultant problem is the high out-of-band spectral density. If the out-of-band signal is filtered off, the reduced PAPR of the clipped signal will regrow [9], [14], [15]. By repeating

\footnotetext{
$\dagger$ Corresponding author

$\ddagger$ This work was supported by National Science Council of R.O.C under grant NSC 92-2213-E-002-075
}

the same procedure several times, both low PAPR and low out-of-band spectral density can be achieved in [16]. Such a method is called recursive clipping and filtering (RCF) in this paper. However, there is still one problem. As the number of recursion increases, although the out-of-band spectral density and the probability of the occurrence of high PAPR decrease, the error rate will increase. The increased error rate is due to the increase of in-band distortion(clipping noise) [2], [16]. With this observation, in this paper, we propose a scheme called recursive clipping and filtering with bounded distortion (RCFBD) to achieve PAPR reduction. Similar to [14], [15], [16], the idea of oversampled digital clipping in the time-domain and removing out-of-band components in the frequency domain is used, but the additional constraint on inband distortion of each tone(subcarrier) is applied during the recursive process. In this way, significant PAPR reduction can be achieved without the penalty of the increased error rate.

In section II, some basics about OFDM, PAPR reduction, and the operation of RCF are provided. Section III describes the system model. Section IV describes the proposed RCFBD. Complexity evaluation is given in section V. Section VI describes the simulation results. Conclusions are given in section VII.

\section{SOME BASICS}

Consider an OFDM system with $N$ subcarriers. Each OFDM block(OFDM symbol), $s(t), 0 \leq t \leq T$, consists of $N$ complex baseband data $X_{0}, X_{1}, \cdots, X_{N-1}$ carried on the $N$ subcarriers respectively for a symbol period of $T$. The OFDM symbol $s(t)$ is

$$
s(t)=\frac{1}{\sqrt{N}} \sum_{k=0}^{N-1} X_{k} e^{j k 2 \pi \Delta f t}, \quad 0 \leq t \leq T
$$

where $\Delta f=1 / T$ is the subcarrier spacing and $X_{k}$ is the complex baseband data modulating the $k$-th subcarrier for $s(t)$. For the OFDM symbol $s(t)$, the peak instantaneous power is

$$
P_{\max }(s(t))=\max _{0 \leq t \leq T}|s(t)|^{2} .
$$

An OFDM symbol sequence can be represented by $\cdots, s(t), s(t+T), \cdots, s(t+m T), \cdots$. We define the average power of the OFDM symbol sequence following the approach in [15], [17] as follows

$$
P_{a v}\left(X_{0}, X_{1}, \cdots, X_{N-1}\right)=\frac{1}{N} \sum_{k=0}^{N-1} E\left[\left|X_{k}\right|^{2}\right]
$$


where $E\left[\left|X_{k}\right|^{2}\right]$ is the expected value of $\left|X_{k}\right|^{2}$. The peak to average power ratio (PAPR) of the OFDM symbol $s(t)$ is

$$
\operatorname{PAPR}(s(t))=\frac{P_{\max }(s(t))}{P_{a v}\left(X_{0}, \cdots, X_{N-1}\right)}
$$

OFDM systems are usually implemented by discrete Fourier transform(DFT). Consider the OFDM signal of (1) sampled at time instant $n \Delta t$, the associated discrete-time output is $s[n]=s(n \Delta t)$. When the signal is sampled by interval $\Delta t=T / L N$, where $L$ is the oversampling factor (OSF). The associated discrete-time output becomes

$$
\begin{aligned}
s_{L}[n]=s\left(n \frac{T}{L N}\right)= & \frac{1}{\sqrt{N}} \sum_{k=0}^{N-1} X_{k} e^{j k 2 \pi \frac{n}{L N}} \\
& \text { for } 0 \leq n \leq L N-1
\end{aligned}
$$

The oversampled signal can be obtained by padding $L N-N$ zeros in the frequency domain and taking the $L N$-point inverse discrete Fourier transform(IDFT). The symbol-wise peak power definition in (2) can be approximated by $P_{\max }(s(t)) \approx$ $\max _{0 \leq n<L N}\left|s_{L}[n]\right|^{2}$ for a large enough $L$.

To alleviate the problem of the occasional occurrence of symbols with high PAPR, many methods have been proposed. Among them, oversampled digital clipping and filtering [14], [15] is a simple and effective method. The basic operations for the oversampled digital clipping and filtering $(\mathrm{OCF})$ are shown in Fig. 1. Complex baseband data $\left(X_{0}, X_{1}, \cdots, X_{N-1}\right)$ are used as input and are converted to time domain data $\left(s_{L}[0]\right.$, $\left.\cdots, s_{L}[N-1], s_{L}[N], \cdots, s_{L}[L N-1]\right)$ by $L N$-point IDFT, which are then clipped by the soft limiter model [9]. The input and output of the soft limiter model are respectively

$$
\text { input : } x=\rho e^{j \phi}, \quad \rho=|x|
$$

and

$$
\text { output : } g(x)= \begin{cases}x, & \text { for } \rho \leq A, \\ A e^{j \phi}, & \text { for } \rho>A .\end{cases}
$$

where $A$ is the clipping threshold. Then $\left(g\left(s_{L}[0]\right), g\left(s_{L}[1]\right), \cdots, g\left(s_{L}[L N-1]\right)\right)$ are converted to $\left(\hat{X}_{0}, \hat{X}_{1}, \cdots, \hat{X}_{N-1}, \cdots, \hat{X}_{L N-1}\right)$ by using the $L N$-point DFT, i.e.,

$$
\begin{aligned}
\hat{X}_{k}= & \frac{\sqrt{N}}{L N} \sum_{n=0}^{L N-1} g\left(s_{L}[n]\right) e^{-j 2 \pi \frac{n k}{L N}} \\
& \text { for } 0 \leq k \leq L N-1
\end{aligned}
$$

The filtering operation removes the out-of-band components and gets $\left(\hat{X}_{0}, \hat{X}_{1}, \cdots, \hat{X}_{N-1}, 0, \cdots, 0\right)$. After the filtering operation, the time domain signal becomes

$$
\begin{aligned}
\hat{s}_{L}[n]= & \frac{1}{\sqrt{N}} \sum_{k=0}^{N-1} \hat{X}_{k} e^{j k 2 \pi \frac{n}{L N}} \\
& \text { for } 0 \leq n \leq L N-1
\end{aligned}
$$

which exhibits the problem of peak power regrowth. That means the peak power $P_{\max }(s(t)) \approx P_{\max }\left(\hat{s}_{L}[n]\right)=$ $\max _{0 \leq n<L N}\left|\hat{s}_{L}[n]\right|^{2}$ will be greater than $A^{2}$ again. The average in-band power following (3) now becomes

$$
P_{a v}\left(\hat{X}_{0}, \cdots, \hat{X}_{N-1}\right)=\frac{1}{N} \sum_{k=0}^{N-1} E\left[\left|\hat{X}_{k}\right|^{2}\right]
$$

which is lower than the original $P_{a v}\left(X_{0}, \cdots, X_{N-1}\right)$ due to the clipping and filtering operation. Since a single round of the OCF operation encounters the problem of peak power regrowth, it is proposed in [16] to repeat the OCF operation several rounds to suppress the final peak power. We call such a method recursive clipping and filtering (RCF).

\section{System MODEL}

The system model considered in this paper consists of two clipping processes. The first is used for PAPR reduction and is called preClip, of which the clipping threshold is denoted as $A$. The second is used to simulate the nonlinearity of the power amplifier by the soft limiter according to (6) and (7). The second clipping is called power amplifier clip(PA-clip) or backoff, of which the clipping threshold is denoted as $A_{a}$. We also use two oversampling factors, i.e, $L$ and $L_{a}$ respectively. $L=2$ is used in the preClip process since it can achieve effective PAPR reduction with the least complexity compared to $L>2 . L_{a}=4$ is used to approximate the analog signal and the nonlinear behavior of the power amplifier in this paper. Although larger $L_{a}$ can achieve more accurate results, $L_{a}=4$ is commonly used to demonstrate the performances of PAPR reduction methods [9], [18].

The performance of a PAPR reduction technique for the OFDM system can be evaluated by the complementary cumulative distribution function (CCDF) of peak power, bit error rate (BER) and out-of-band power spectral density (PSD). Here, the CCDF of peak power is used instead of the conventional CCDF of PAPR, since power amplifier is peakpower-limited and the average power may vary for various PAPR reduction techniques. Consider the 128-tone OFDM $\operatorname{system}(N=128)$ having unit average symbol energy, i.e. $E\left[\left|X_{k}\right|^{2}\right]=1, k=1, \cdots, 128$. Simulation results using RCF scheme, including the CCDF of peak power, BER and PSD, are given in Fig. 2, 3 and 4 respectively for 16-QAM signal constellation, where RCF- $J, J=1,2,4,8$, indicates RCF with $J$ recursions. In the simulation, $A$ and $A_{a}$ are both set to be equal to 1.413 , which corresponds to $3 \mathrm{~dB}$ power backoff when the input average power is 1 . It is observed that while the the number of recursions increases, the out-of-band PSD and the probability of the occurrence of high PAPR decrease but the error rate increases. The drawback of the increased error rate is due to the increased distortion caused by the recursive clipping operations. In the following section, we propose a method to restrict the distortion.

\section{Recursive ClitPing AND FILTERING With BOUNDED DISTORTION}

The introducing of bounded distortion(BD) comes from the idea of constellation perturbation(distorted approach) as compared to the existing constellation extension(distortionless approach) [9], [11]. When the power amplifier is operated with small backoff, the distorionless approach will still face the clipping distortion due to the insufficient PAPR reduction. The idea of constellation perturbation is to disturb the signal points on all tones under the constraint of the preset bound in order to reduce PAPR while keeping the impact on BER under control. With the help of RCF, we can use OCF to 
determine how the signal point on each tone is disturbed at each recursion, then we can impose $\mathrm{BD}$ control to restrict the range of the perturbation.

Consider the scheme of a single operation of clipping and filtering with bounded distortion control(BD control) as shown in the dashed box of Fig. 5. This scheme is simply composed of the OCF operation followed by an operation of BD control. The input and output of the OCF operation are $\left(X_{0}, X_{1}, \cdots, X_{N-1}\right)$ and $\left(\hat{X}_{0}, \hat{X}_{1}, \cdots, \hat{X}_{N-1}\right)$ respectively. The operation of BD control has input $\left(\hat{X}_{0}, \hat{X}_{1}, \cdots, \hat{X}_{N-1}\right)$ and output $\left(\tilde{X}_{0}, \tilde{X}_{1}, \cdots, \tilde{X}_{N-1}\right)$ respectively, in addition to the reference $\left(X_{0}, X_{1}, \cdots, X_{N-1}\right)$ and distortion bound $\delta$. The goal of BD control is to adjust each $\hat{X}_{k}, k=0,1, \cdots, N-1$, which comes from the OCF operation, so that the adjusted resultant value $\tilde{X}_{k}$ falls in a region determined by the reference signal point $X_{k}$ and the preset bound $\delta$.

For an $M-Q A M$ OFDM system with constellation size $M$ being a square of even integer and having average energy 1, the algorithm of BD control is as follows.

$$
\begin{aligned}
& \text { BD control for square M-QAM } \\
& \star \text { Input : } \hat{X}_{k}=(\hat{a}, \hat{b}) \\
& \star \text { Output : } \tilde{X}_{k}=(\tilde{a}, \tilde{b}) \\
& \text { * Reference : } X_{k}=(a, b) ; a, b \in\left\{\frac{i}{\sqrt{2(M-1) / 3}}, i=\right. \\
& \pm 1, \pm 3, \cdots, \pm(\sqrt{M}-1)\} \\
& \Delta x=\hat{a}-a, \Delta y=\hat{b}-b, \gamma=\frac{\sqrt{M}-2}{\sqrt{2(M-1) / 3}} ; \\
& \text { if }(|\Delta x| \leq \delta) \quad \tilde{a}=\hat{a} \text {; } \\
& \text { elseif }((a>0 \text { and } \Delta x<0) \text { or }(a<0 \text { and } \Delta x>0) \text { or }(|a|<\gamma)) \\
& \tilde{a}=a+\operatorname{sign}(\Delta x) \delta \\
& \text { else } \\
& \tilde{a}=\hat{a} \text {; } \\
& \text { if }(|\Delta y| \leq \delta) \quad \tilde{b}=\hat{b} \text {; } \\
& \text { elseif }((b>0 \text { and } \Delta y<0) \text { or }(b<0 \text { and } \Delta y>0) \text { or }(|b|<\gamma)) \\
& \tilde{b}=b+\operatorname{sign}(\Delta y) \delta
\end{aligned}
$$

else

$$
\tilde{b}=\hat{b} \text {; }
$$

where $\operatorname{sign}(z) \in\{+,-\}$ is the sign of $z$ and $\gamma$ is the threshold for determining whether the real part or imaginary part of a reference signal point is on the boundary of the $M-Q A M$ constellation or not. Note that for $M-Q A M$ OFDM systems, in case that the real part $a$ of the reference signal point is located on the boundary of the constellation and the real part $\hat{a}$ of the distorted signal point $\hat{X}_{k}$ is outside the boundary and can enhance the capability against noise as compared to the reference signal point, it is not required that $\hat{a}$ be restricted in the region $[a-\delta, a+\delta]$. The release of the constraint for the imaginary part is applied in the same manner and is independent of the real part processing. The desired regions of output signal points of BD control for 16- $Q A M$ constellation is shown in Fig. 6 respectively.

A single operation of OCF with BD control can not achieve satisfactory PAPR reduction and BER simultaneously in general. We need to repeat the same operation several times. We call such a scheme recursive clipping and filtering with bounded distortion (RCFBD). RCFBD operates in a manner similar to RCF, while guaranteeing that the distortion be bounded and providing more freedom in achieving PAPR reduction and low BER. The distortion bound and clipping threshold used at the $j$ th recursion are denoted as $\delta^{(j)}$ and $A^{(j)}, 0 \leq j<J$ respectively. The operation of RCFBD- $J$ is described as follows.

\section{RCFBD- $J$}

$\star$ Input : $\left(X_{0}, X_{1}, \cdots, X_{N-1}\right)$

$\star$ Output : $\left(\tilde{X}_{0}, \tilde{X}_{1}, \cdots, \tilde{X}_{N-1}\right)$

$\star$ Reference of BD control : $\left(X_{0}, X_{1}, \cdots, X_{N-1}\right)$

$\star$ Number of Recursions: $J$

1) Set $j=0$;

$$
\text { Set } X_{k}^{(0)}=X_{k} \text {, for } 0 \leq k \leq N-1 \text {; }
$$

2) While $(j<J)\{$

Use $\left(X_{0}^{(j)}, X_{1}^{(j)}, \cdots, X_{N-1}^{(j)}\right)$ as input to the operation of OCF with clipping threshold $A^{(j)}$ and achieve output $\left(\hat{X}_{0}^{(j)}, \hat{X}_{1}^{(j)}, \cdots, \hat{X}_{N-1}^{(j)}\right)$;

Use $\left(\hat{X}_{0}^{(j)}, \hat{X}_{1}^{(j)}, \cdots, \hat{X}_{N-1}^{(j)}\right)$ as input to the operation of BD control with distortion bound $\delta^{(j)}$ and achieve output $\left(\tilde{X}_{0}^{(j)}, \tilde{X}_{1}^{(j)}, \cdots, \tilde{X}_{N-1}^{(j)}\right)$;

Set

$\left(X_{0}^{(j+1)}, X_{1}^{(j+1)}, \cdots, X_{N-1}^{(j+1)}\right)=\left(\tilde{X}_{0}^{(j)}, \tilde{X}_{1}^{(j)}, \cdots, \tilde{X}_{N-1}^{(j)}\right)$; Set $j=j+1$;

\}

3) The final output is $\left(\tilde{X}_{0}, \tilde{X}_{1}, \cdots, \tilde{X}_{N-1}\right)$ = $\left(\tilde{X}_{0}^{(J-1)}, \tilde{X}_{1}^{(J-1)}, \cdots, \tilde{X}_{N-1}^{(J-1)}\right)$. This is the data block to be sent for OFDM transmission satisfying the bounded distortion constraint and having reduced PAPR.

The process of RCFBD is illustrated in Fig. 5. During the recursive process of RCFBD, there will be benefit by properly varying the distortion bound and the clipping threshold applied at each recursion when large $\delta$ is used. In addition to the case of constant bounds/thresholds $\left(A^{(j)}=A, \delta^{(j)}=\delta\right.$, for $0 \leq j<$ $J$ ), we consider an OFDM system using RCFBD- $J$ scheme as follows of which the distortion bound is exponentially decreasing and the clipping threshold is linearly increasing as the number of recursions increases.

Varying Distortion Bounds and Clipping Thresholds

$$
\begin{aligned}
& \delta^{(j)}=\left\{\begin{array}{c}
\alpha \delta e^{(-\beta j)}, \text { for } 0 \leq j<\lfloor\epsilon J\rfloor \\
\delta, \quad \text { for }\lfloor\epsilon J\rfloor \leq j<J
\end{array}\right. \\
& A^{(j)}=A^{(0)}+\frac{\left(A-A^{(0)}\right) j}{J}, \text { for } 0 \leq j<J,
\end{aligned}
$$

There are several parmaters such as $A^{(0)}, \eta, \beta$ and $\epsilon$ which are chosen through simulations. The idea of this design is that the beginning clipping threshold $A^{(0)}$ is chosen to be sufficiently smaller than the final clipping threshold $A$ and $\delta^{(0)}$ is larger than the preset $\delta$ so that more distortion occurs at the first recursion. As the number of recursion increases, 
$\delta^{(j)}$ is gradually reduced and $A^{(j)}$ is gradually increased to achieve a better solution of low peak power and low error rate.

\section{COMPLEXITy OF RCFBD SCHEME}

Each recursion of RCFBD takes one IFFT(inverse fast Fourier transform), one digital clipping, one FFT(fast Fourier transform) and one BD control. One IFFT or FFT with $O S F=L$, i.e. $L N$-point transform, takes $(L N / 2) \log _{2}(L N)$ complex multiplications and $(L N) \log _{2}(L N)$ complex number additions [19]; one digital clipping operation with $O S F=L$ takes $L N$ complex multiplications to compute the power of the $L N$ time domain samples and compare with the clipping power threshold, then clip the samples when necessary. Since only a small fraction of samples are clipped, the complexity of digital clipping is negligible as compared to FFT. Each BD control takes $2 L N$ real number additions. Hence, the computational complexity is dominated by IFFT/FFT for each recursion of RCFBD and can be roughly estimated as the complexity of two $L N$-point FFT operations. The recursion times, $J$, determines the total computational complexity of RCFBD- $J$, which is equivalent to $2 J$ FFT operations.

In this paper, we consider RCFBD- $J$, where $J=8$ recursions are needed and oversampling factor $L=2$ is used in the recursive process for PAPR reduction. Hence, the complexity of PAPR reduction of RCFBD-8 is approximately equal to that of 16 FFT operations.

\section{Simulation Results and Discussion}

Simulation results of 16-QAM/128-tone OFDM systems for RCFBD- $J$ with recursion times $J=8$ under additive white gaussian noise(AWGN) channel and 3dB PA-clip $\left(A_{a}=1.413\right)$ are presented in 2, 3 and 4 respectively. The preset distortion bounds $\delta=\frac{0.7}{\sqrt{10}}$ and $\delta=\frac{0.8}{\sqrt{10}}$ are considered. Both constant and varying distortion bounds/clipping thresholds are simulated. It is seen that RCFBD with varying $\delta^{(j)}$ and $A^{(j)}$ can be superior to RCFBD with constant $\delta^{(j)}$ and $A^{(j)}$. The parameters used for the case of varying bounds and thresholds are $A=1.413(3 \mathrm{~dB}), A^{(0)}=1.230(1.8 \mathrm{~dB}), \eta=4.0, \beta=0.38$, and $\epsilon=0.75$. The parameters used for the case of constant bounds and thresholds are $A=1.39$ ( which is actually the value of $A^{(J-1)}$ of the varying case following (11) ). We see that RCFBD has PAPR reduction similar to RCF- $J, J \geq 2$, while achieving lower BER, especially at high signal-to-noise ratio conditions. Simulation results for the 16-QAM RCFBD with varying $\delta^{(j)}$ and $A^{(j)}$ using $\delta=\frac{0.3}{\sqrt{10}}$ and $\delta=\frac{0.5}{\sqrt{10}}$ are also provided to demonstrate the performance trend with respect to $\delta$. We see that $\delta=\frac{0.5}{\sqrt{10}}$ yield the best BER when SNR is larger than $17.5 \mathrm{~dB}$.

Note that the average power of the ideal case is 1 while the average power of the original case is reduced to 0.865 due to the clipping by power amplifier. Either RCF- $J$ or RCFBD- $J$ yields lower average power as $J$ increases. Moreover, RCFBD has lower average power than RCF. Let $V_{1}, V_{2}, \cdots, V_{M}$ denote the $M$ signal points of the signal constellation. Suppose that many OFDM symbols are transmitted, the mean of all the distorted data which are originally represented by the signal point $V_{i}$ will have its amplitude smaller than $\left|V_{i}\right|$. The phenomenon is called constellation shrinkage [20], [21]. The BER results for RCF- $J$ shown in Fig. 3 are obtained by considering the effect of constellation shrinkage in the detection, which is implemented via dividing the received signal by the shrinking factor. The shrinking factor is estimated as the square root of the average power after PA-clip. However, the BER results for RCFBD shown in Fig. 3 are obtained without considering the effect of constellation shrinkage in the detection. Although constellation shrinkage also occurs to RCFBD, simulation results indicate that it is of no benefit to take constellation shrinkage into account for RCFBD.

\section{CONCLUSIONS}

The proposed RCFBD scheme achieves significant PAPR reduction while keeping the distortion of the data carried on each subcarrier under control. Such a scheme eliminates the need of the side information and simplifies the work of the corresponding OFDM receiver. Compared to RCF, RCFBD provides more tunable parameters for achieving better PAPR reduction, out-of-band emission and BER performance. The simulation results show that RCFBD is more robust against AWGN noise than RCF while achieving similar PAPR reduction and lower out-of-band emission.

\section{REFERENCES}

[1] J. Bingham, "Multicarrier modulation for data transmission: An idea whose time has come," IEEE Commun. Mag., vol. 28, pp. 5-14, May 1990.

[2] X. Li and L. C. Jr., "Effects of clipping and filtering on the performance of OFDM,' Proc. VTC'97, May 1997.

[3] R. O'Neill and L. Lopes, "Envelope variations and spectral splatter in clipped multicarrier signals," Proc. PIMRC '95, vol. 1, Sept. 1995.

[4] A. Jones and T. Wilkinson, "Combined coding for error control and increased robustness to system nonlinearities in OFDM," Vehicular Technology Conference, vol. 2, May 1996.

[5] K.Sathananthan and C. Tellambura, "Coding to reduce both PAR and PICR of an OFDM signal," IEEE Commun. Letters, vol. 6, pp. 316-318, Aug. 2002.

[6] R. Bauml, R. Fischer, and J. Huber, "Reducing the peak-to-average power ratio of multicarrier modulation by selected mapping," Electronic Letters, vol. 32, pp. 2056-2057, 1996.

[7] J. Eetvelt, G.Wade, and M. Tomlinson, "Peak to average power reduction for OFDM schemes by selective scrambling," Electronic Letters, vol. 32, pp. 1963-1964, 1996.

[8] C. Tellambura, "Use of m-sequences for OFDM peak-to-average power ratio reduction," Electronic Letters, vol. 33, pp. 1963-1964, July 1997.

[9] J. Tellado, "Multicarrier modulation with low PAR - applications to dsl and wireless," Jan. 2000.

[10] B. Krongold and D. Jones, "An active-set approach for ofdm par reduction via tone reservation," IEEE Trans. Signal Processing, vol. 52, pp. 495-509, Feb. 2004.

[11] B. Krongold and D. Jones, "Par reduction in ofdm via active constellation extension," IEEE Trans. Broadcasting, vol. 49, pp. 258-268, Sept. 2003.

[12] I. Tasadduq and R. Rao, "PAPR reduction of OFDM signals using multiamplitude CPM," Electronic Letters, vol. 38, Aug. 2002.

[13] D. Wulich and Glinowiecki, "Level clipped high-order ofdm," IEEE Trans. Commun., vol. 48, pp. 928-930, June 2000.

[14] J.Armstrong, "New OFDM peak-to-average power reduction scheme," Vehicular Technology Conference, May 2001.

[15] H. Ochiai and H. Imai, "Performance of deliberate clipped OFDM signals," IEEE Trans. Commun., vol. 50, Jan. 2002.

[16] J.Armstrong, "Peak-to-average power ratio reduction for OFDM by repeated clipping and frequency domain filtering," Electronic Letters, vol. 38 , Feb. 2002 
[17] G. Wunder and H. Boche, "Peak value estimation of band-limited signals from its samples, noise enhancement, and a local characterization," IEEE Trans. Signal Processing.

[18] A. Jayalath and C. Athaudage, "On the PAR reduction of OFDM signals using multiple signal representation," Proc. IEEE PIMRC'03, 2003.

[19] A. Oppenheim and R. Schafer, "Discrete-time signal processing," 1989.

[20] D. Dardari, V. Tralli, and A. Vaccari, "A theoretical characterization of nonlinear distortion effects in ofdm systems," IEEE Trans. Communications, vol. 48, pp. 1755 - 1764, Oct. 2000.

[21] K. Panta and J. Armstrong, "Effects of clipping on the error performance of OFDM in frequency selective fading channels," IEEE Trans. Wireless Commun., vol. 3, pp. 668 - 671, Mar. 2004.
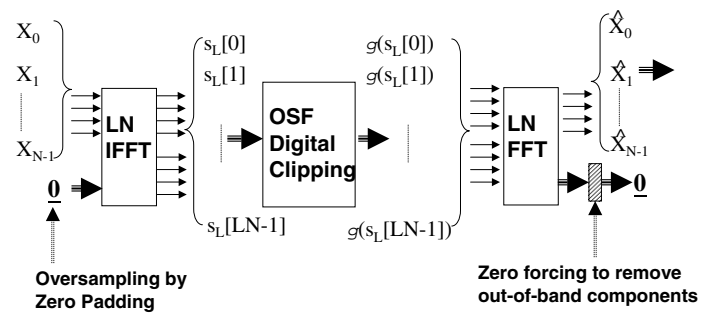

$\mathrm{N}$ is the number of tones

OSF is the overampling factor, OSF $=\mathrm{L}$

Fig. 1. Oversampled Clipping and Filtering

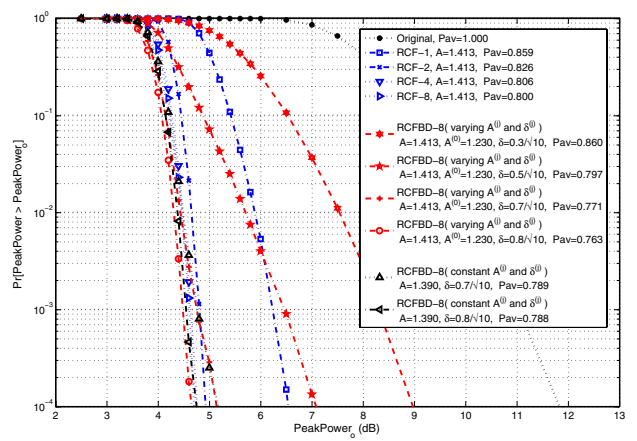

Fig. 2. Complementary Cumulative Distribution Function(CCDF) of Peak Power for 16-QAM/128-OFDM

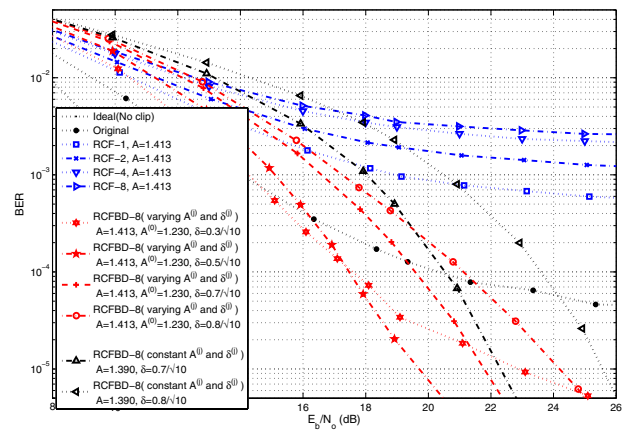

Fig. 3. Bit Error Rate(BER) under 3dB Backoff for 16-QAM/128-OFDM

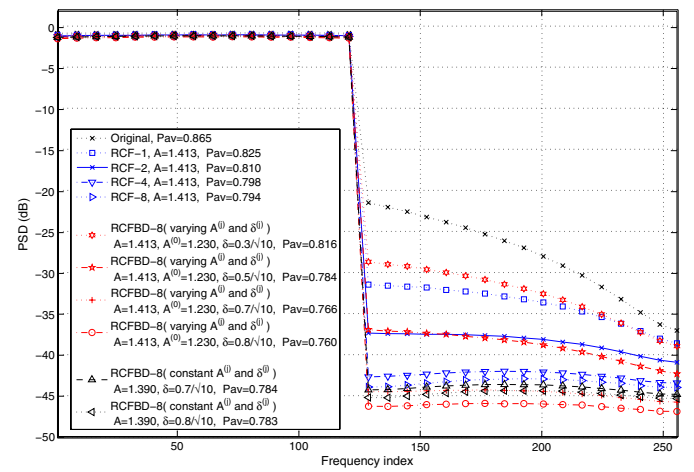

Fig. 4. Power Spectral Density(PSD) under 3dB Backoff for 16-QAM/128-OFDM

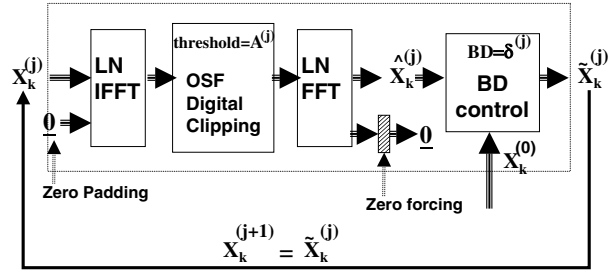

Fig. 5. Recursive Clipping and Filtering with Bounded Distortion(RCFBD)

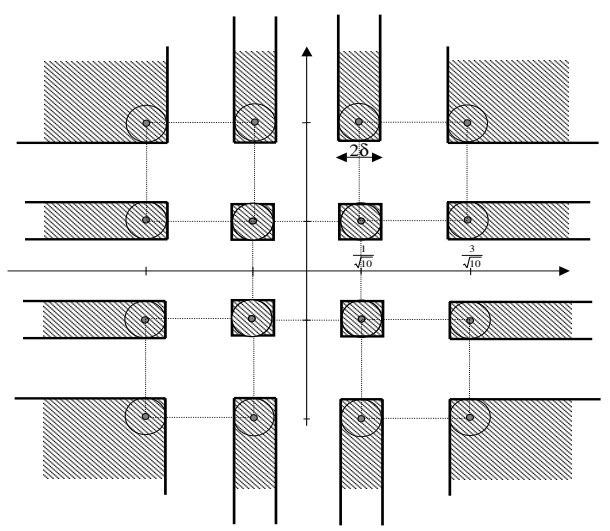

Fig. 6. Regions of Bounded Distortion control for 16-QAM 